\title{
Eqüidade na atenção pré-natal e ao parto em área da Região Metropolitana de São Paulo, 1996
}

\author{
Prenatal and childbirth care in an area \\ in Greater Metropolitan São Paulo, 1996
}

Rosana Fiorini Puccini 1

Glaura César Pedroso 1

Edina Mariko Koga da Silva 1

Norma Sueli de Araújo 2

Nilza Nunes da Silva 3

\footnotetext{
1 Departamento de Pediatria, Universidade Federal de São Paulo. Rua Botucatu 598, São Paulo, SP 04023-062, Brasil. 2 Secretaria Municipal de Saúde. Av. Elias Yazbeq 3088, Embu, SP 06801-970, Brasil.

3 Departamento de Epidemiologia, Faculdade de Saúde Pública, Universidade de São Paulo, Av. Dr. Arnaldo 715, São Paulo, SP 01246-904, Brasil.
}

\begin{abstract}
A cross-sectional study was conducted to evaluate prenatal and childbirth care interviewing mothers of infants in the municipality of Embu (Greater Metropolitan São Paulo) in 1996, according to four socioeconomic strata. A door-to-door survey included a probabilistic sample consisting of 483 infants. In all strata more than 90\% of the mothers had received prenatal care, but with late access in stratum 4 (residents of favelas, or slums). Breast examination during prenatal care, reported by only $60.8 \%$ of the mothers, was the worst single indicator of quality of prenatal care in the municipality. The outcome indicator - first prenatal consultation after the first trimester and total number of consultations less than six-was associated with maternal age (less than 20 years), low per capita family income (less than one minimum wage), and lack of private health plan. As for deliveries, 97.7\% occurred in hospital, of which 32.5\% by cesarean section, with the latter more frequent in private health care facilities (63.2\%). No population segments were identified as being excluded from the health care system, but some indicators suggest greater deficiencies in socioeconomic stratum 4. These results have supported local health system managers in redefining health measures for the municipality.
\end{abstract}

Key words Equity; Local Health Systems; Prenatal Care; Cross-Sectional Studies

Resumo Estudo transversal realizado com o objetivo de avaliar a assistência pré-natal e ao parto de mães de crianças menores de um ano residentes no Município do Embu (Região Metropolitana de São Paulo), no ano de 1996, segundo quatro estratos de condições de vida. Inquérito domiciliar considerou uma amostra probabilística constituída por 483 crianças. Em todos os estratos, mais de 90\% das mães realizaram pré-natal, porém com acesso tardio no estrato 4 (favelas). A realização do exame de mamas, isoladamente, foi o pior indicador de qualidade da assistência pré-natal do município, referida por apenas 60,8\% das mães. O desfecho - início do prénatal após o 1o trimestre e o número de consultas inferior a seis - associou-se à idade materna (menor de 20 anos), menor renda (inferior a 1 salário mínimo per capita) e à falta de acesso a plano privado de saúde. Quanto aos partos, 97,7\% deles foram hospitalares; houve 32,5\% de cesarianas, que foram mais freqüentes nos serviços privados (63,2\%). Não se identificaram segmentos populacionais excluídos do sistema de saúde, no entanto, alguns indicadores apontaram para deficiências mais acentuadas no estrato 4. Estes resultados têm subsidiado os gestores locais na definição de ações de saúde para o município.

Palavras-chave Eqüidade; Sistemas Locais de Saúde; Cuidado Pré-Natal; Estudos Transversais 


\section{Introdução}

Ainda que esteja bem comprovado o papel de determinantes sócio-econômicos na saúde da criança e da mulher, vários estudos demonstram a importância do acesso e qualidade da assistência à saúde na evolução favorável dos principais indicadores populacionais de saúde verificada em nosso país, mesmo diante de crises econômicas, desemprego e persistência de inadequadas condições ambientais e de moradia de um grande contingente da população (Monteiro et al., 2000a, 2000b). Em alguns casos, acentuaram-se desigualdades regionais, revelando a persistência de diferenças profundas, não apenas no acesso aos serviços de saúde, mas na qualidade e adequação dos mesmos (Fonseca \& Laurenti, 2000; Monteiro et al., 2000b).

A assistência pré-natal compreende um conjunto de atividades que visa à promoção da saúde da mulher grávida e do feto, bem como a identificação de riscos, para ambos, visando à assistência adequada e oportuna. Sua ausência e/ou deficiência, comprovadamente, associamse a maiores taxas de morbi-mortalidade neonatal, prematuridade, baixo peso ao nascer, retardo de crescimento intra-uterino e mortalidade materna (Barbieri et al., 2000, Bolzán et al., 2000; Fonseca \& Laurenti, 2000; Kramer, 1987; Menezes et al., 1998; Monteiro et al., 2000c; Schlaepfer \& Infante, 1995).

Entre os indicadores de qualidade da assistência pré-natal, o Ministério da Saúde (MS, 2000) inclui a taxa de cobertura do programa, realização de seis ou mais consultas de pré-natal, início no primeiro trimestre de gestação e realização de exames laboratoriais. Dados referentes a 1996 (MS, 1997) demonstravam que, no país, apenas 52,9\% das mães de recém-nascidos vivos realizaram seis ou mais consultas de pré-natal. No Estado de São Paulo e Região Metropolitana, no mesmo ano, estas freqüências foram de $61,5 \%$ e $54,5 \%$, respectivamente. Quanto ao início do pré-natal, no Nordeste, 51,9\% das gestantes realizaram a primeira consulta no primeiro trimestre de gestação e, na Região Sul, esta porcentagem foi de $79,7 \%$.

No Embu, nos anos de 1993 a 1996 - período correspondente à administração municipal que integrou a equipe deste estudo -, a cobertura da assistência pré-natal, calculada por meio de estimativas populacionais e de dados de produção, indicavam que cerca de $50 \%$ das gestantes realizavam pré-natal na Rede Básica do Município (SMS-Embu, 1996); informações obtidas por ocasião da primeira consulta de crianças matriculadas nas unidades básicas do município, ao contrário, indicavam que a maioria das mães havia realizado pré-natal; contudo, parte delas em serviços externos ao Embu. Não havia leitos de maternidade no município, na ocasião, e as informações referentes ao parto, obtidas também na consulta da criança, indicavam que este era quase sempre hospitalar. Os coeficientes de mortalidade infantil e materna apresentavam uma tendência de declínio; entretanto, mantinham-se mais elevados que os do Município e Estado de São Paulo (Fundação Seade, 2001).

A ausência de informações precisas referentes à assistência pré-natal e ao parto e a possibilidade de que segmentos da população se encontrassem excluídos dessa assistência motivaram a realização deste trabalho, que teve como objetivo principal descrever a assistência prénatal e ao parto das mães de crianças menores de um ano residentes no Município do Embu, no ano de 1996, segundo estratos de condições de vida, bem como identificar possíveis fatores associados à ausência ou inadequação dessa assistência.

\section{Metodologia}

O presente estudo é parte integrante do projeto Morbidade Infantil e Utilização de Serviços de Saúde, financiado pela Fundação de Amparo à Pesquisa do Estado de São Paulo (FAPESP), que envolveu pesquisadores e profissionais da Faculdade de Saúde Pública da Universidade de São Paulo (USP), da Universidade Federal de São Paulo (UNIFESP) e da Prefeitura Municipal da Estância Turística de Embu, no ano de 1996 (Silva et al., 1997).

\section{Caracterização do Município do Embu e a assistência pré-natal}

O Município do Embu compõe a Sub-Região Sudoeste da Região Metropolitana de São Paulo (RMSP), caracterizada por possuir pequena concentração industrial, baixo potencial de crescimento econômico e por situar-se em amplas áreas de proteção de mananciais (EMPLASA, 1994). O Embu é uma estância turística completamente urbanizado e sua população, em 1996, era de 194.879 habitantes. Nesse mesmo ano, o Coeficiente de Mortalidade Infantil foi de 28,12 por mil nascidos vivos, e o componente neonatal correspondia a cerca de dois terços desse Coeficiente (Fundação Seade, 2001).

Em 1996, a Rede Municipal de Saúde estava constituída por nove Unidades Básicas de Saúde (UBS) e dois prontos-socorros (PS). Não havia leitos hospitalares na época da realização 
deste estudo. O Programa de Integração Docente-assistencial da UNIFESP (PIDA-Embu), em 1996, envolvia os departamentos/disciplinas de Pediatria, Neurologia Infantil, Psiquiatria, Gineco-obstetrícia, Fonoaudiologia e Tecnologia Oftálmica. Além de atividades de ensino, assistência e pesquisa, a universidade mantém uma participação ativa no Conselho Municipal de Saúde, desde 1991 (UNIFESP, 2001).

Em 1996, teve início uma reestruturação da assistência pré-natal no Embu que incluiu: educação permanente aos profissionais que atuavam no pré-natal (médicos e enfermeiros), definição de um cronograma de consultas e normalização de condutas diagnósticas e terapêuticas, conforme critérios de risco gestacionais, criação dos serviços de ultra-sonografia, tococardiografia e pré-natal patológico, este dirigido a gestantes portadoras de doenças crônicas, tendo-se conseguido garantia de referência hospitalar para estes casos. Embora representasse um avanço na organização do serviço, a maioria das gestantes permanecia sem acesso referenciado à maternidade (SMS-Embu, 1996).

\section{- População de estudo}

Crianças menores de um ano e suas mães. A coleta de dados, no domicílio, foi realizada no período de agosto a novembro de 1996. Para análise da assistência pré-natal, foram considerados apenas os questionários respondidos pela mãe.

\section{- Estratos populacionais de condições de vida}

Dispondo de arquivos de dados do Censo Demográfico de 1991 (IBGE, 1992), a população foi estratificada em agrupamentos homogêneos definidos de acordo com condições de moradia e situação sócio-econômica da família. Os 135 setores censitários do município foram reunidos por intermédio da técnica de análise de agrupamentos denominada average linkage (Frei, 1997). O tipo de moradia, o número de moradores, saneamento básico, a renda e a escolaridade dos chefes de família foram as variáveis consideradas para discriminar os quatro estratos resultantes:

a) Estrato 1: constituído por 17 setores censitários concentrados na área turística do município; apresentava melhores condições de ambiente, renda, moradia e escolaridade.

b) Estratos 2 e 3: constituídos por 67 e 34 setores censitários, respectivamente; refletiam as condições ambientais predominantes no município. Foram mantidos separados devido à presença de maior número de favelas no estrato 3. c) Estrato 4: constituído por 17 setores censitários, todas as moradias foram classificadas como aglomerados subnormais (favelas), segundo o Censo Demográfico de 1991. As condições ambientais, de moradia, renda e escolaridade eram as piores do município.

\section{- Processo de amostragem}

O processo de sorteio adotado foi o de conglomerados em dois estágios. No primeiro, $10 \mathrm{se}$ tores censitários foram sorteados, em cada estrato, pelo critério de partilha proporcional aos seus tamanhos (Kish, 1965), considerando-se o número de domicílios registrados no Censo Demográfico de 1991. No segundo estágio, sortearam-se domicílios dentro de cada setor anteriormente selecionado, após atualização do número de domicílios existentes. A amostra final foi constituída por 483 crianças menores de um ano e 475 famílias. Havia quatorze crianças gemelares e uma família apresentava duas crianças menores de um ano; todavia, uma delas não tinha grau de parentesco com essa família. Na descrição da assistência pré-natal e ao parto, foram consideradas 475 gestações; na descrição das intercorrências neonatais e do peso ao nascer foram consideradas 483 crianças; variáveis com mais de $20 \%$ de perda de informação não foram analisadas. A distribuição das crianças, segundo os estratos de condições de vida, foi a seguinte: estrato 1: 80 crianças; estrato 2: 138 crianças; estrato 3: 116 crianças; estrato 4: 149 crianças.

\section{- Variáveis analisadas}

a) Pré-natal adequado: realização de seis ou mais consultas, início no primeiro trimestre de gestação, realização de exames laboratoriais, aferição de pressão arterial (em uma ou mais consultas), exame das mamas (em uma ou mais consultas) e realização de pelo menos uma ultrasonografia (este exame compunha a lista de procedimentos do Programa de Assistência Pré-natal do Município). Foi considerado pré-natal não adequado se um ou mais itens estivessem ausentes. Para a análise multivariada considerouse apenas o número de consultas inferior a seis, com início após o primeiro trimestre, pois julgamos que os demais indicadores teriam outros determinantes, incluindo a atuação do profissional. b) Assistência ao parto: hospital (nome e município), tipo de parto, peso ao nascer, intercorrências neonatais (considerou-se como intercorrência neonatal presente quando referida na entrevista e/ou quando a alta do berçário ocorreu após cinco dias de vida). 


\section{- Análise estatística}

Para processamento e análise dos dados, utilizou-se o programa Epi Info - versão 6.04, incluindo o módulo CSAMPLE que permite ponderação dos mesmos. A amostragem por conglomerados introduziu diferenças de probabilidade $(f)$ de qualquer família ou criança da população pertencer à amostra sorteada. Para cada estrato o peso $w$ foi calculado pelo inverso da probabilidade $f$. Ou seja, $w=1 / f$. A expressão matemática adotada para calcular $f$ decorreu do plano de amostragem adotado (Silva et al., 1997). A significância estatística foi feita considerando as estimativas, erros-padrão e intervalos de confiança (95\%).

Para análise multivariada, levou-se em conta o desfecho, início do pré-natal após o 1o trimestre e número de consultas menor do seis; foi utilizado o modelo de regressão logística e analisadas as variáveis que alcançaram valor calculado de p menor ou igual a $20 \%$. Para esta análise, utilizou-se o programa estatístico MULTLR. Em todos os testes estatísticos, adotou-se o nível de significância de 5\% (alfa = 0,05).

\section{Resultados}

Na Tabela 1, estão apresentados dados sócioeconômicos e demográficos das famílias das crianças da amostra, consoante os quatro estratos de condições de vida. Mesmo sem significância estatística, evidencia-se uma tendência de piores condições, no estrato 4, quanto à renda, escolaridade e alfabetização dos pais e história de óbitos infantis; a maior estabilidade das famílias do estrato 1 reflete-se na freqüência de mães e pais que residem há mais de cinco anos ou são naturais do Embu; o menor acesso a plano privado de saúde no estrato 4 apresentou diferença estatisticamente significante com relação aos demais estratos e ao total do município. Na Tabela 2, podemos observar que mais de $90 \%$ das mães haviam realizado pré-natal não se observando diferenças entre os estratos. A realização de pré-natal na Rede Básica de Saúde do Embu foi pouco mais freqüente nos estratos 1 e 4 (diferença sem significância estatística). Quanto aos indicadores de qualidade do pré-natal, há deficiências importantes em todos os estratos; tais deficiências, entretanto, são mais acentuadas quanto ao início no lo trimestre, número de consultas e exame de mamas; houve diferença estatisticamente significante entre o estrato 1 e 4 quanto ao início do pré-natal, sendo mais desfavorável no estrato 4 . $\mathrm{O}$ indicador - pré-natal adequado - mostrou-se com baixa freqüência em todos os estratos, com tendência decrescente do estrato 1 ao 4 (sem diferenças estatisticamente significantes).

Na Tabela 3, verificamos que o parto hospitalar foi quase universal. A freqüência de cesarianas não apresentou diferenças estatisticamente significantes entre os estratos, embora com porcentagens pouco mais elevadas nos estratos 1 e 3. Na mesma tabela, observamos que no estrato 1 , os hospitais públicos e os privados/convênios foram mais utilizados; os privados conveniados com o SUS foram menos utilizados no estrato 1, quando comparados aos estratos 2, 4 e total do município; no estrato 4, os hospitais privados/convênio foram menos utilizados (diferenças estatisticamente significantes em relação ao estrato 1 e total do município). A freqüência de intercorrências neonatais apresentou uma tendência crescente do estrato 1 ao 4 . Esta diferença foi estatisticamente significante entre os estratos 1 e 4 . As intercorrências neonatais mais freqüentes foram: infecções pulmonares e outras $(13,5 \%)$, hipoglicemia $(13,5 \%)$, sofrimento fetal $(12,2, \%)$, prematuridade $(9,5 \%)$ e o desconforto respiratório $(8,1 \%)$. Ainda na Tabela 3, nota-se que não existiu diferença no que se refere ao peso de nascimento.

Quanto ao local de nascimento, 68,4\% dos partos ocorreram em São Paulo, 19,7\% em outros municípios da Região Metropolitana, 1,7\% no Embu e os demais no interior de São Paulo ou outros estados. As freqüências de cesarianas, segundo o tipo de serviço, apresentaram diferenças estatisticamente significantes: nos serviços privados, a proporção de cesarianas foi de 63,2\% (IC: 51,4-75,1), nos serviços próprios do SUS foi de $32,9 \%$ (IC: $24,3-41,5$ ), e nos serviços privados conveniados com o SUS foi de 13,5\% (IC: 1,9-25,1).

Na Tabela 4, verificamos que o pré-natal adequado, de acordo com os critérios estabelecidos neste estudo, foi mais freqüente na Rede Básica do Embu em comparação à assistência realizada fora do município (sem diferença estatisticamente significante). Os resultados da análise multivariada encontram-se na Tabela 5. O desfecho - início do pré-natal após o primeiro trimestre e número de consultas inferior a seis esteve associado à idade materna (menor de 20 anos), à renda (menor que um salário mínimo per capita) e à falta de acesso a plano privado de saúde. 
Variáveis sócio-demográficas das famílias $(n=475)$ de crianças menores de um ano. Embu, São Paulo, Brasil, 1996.

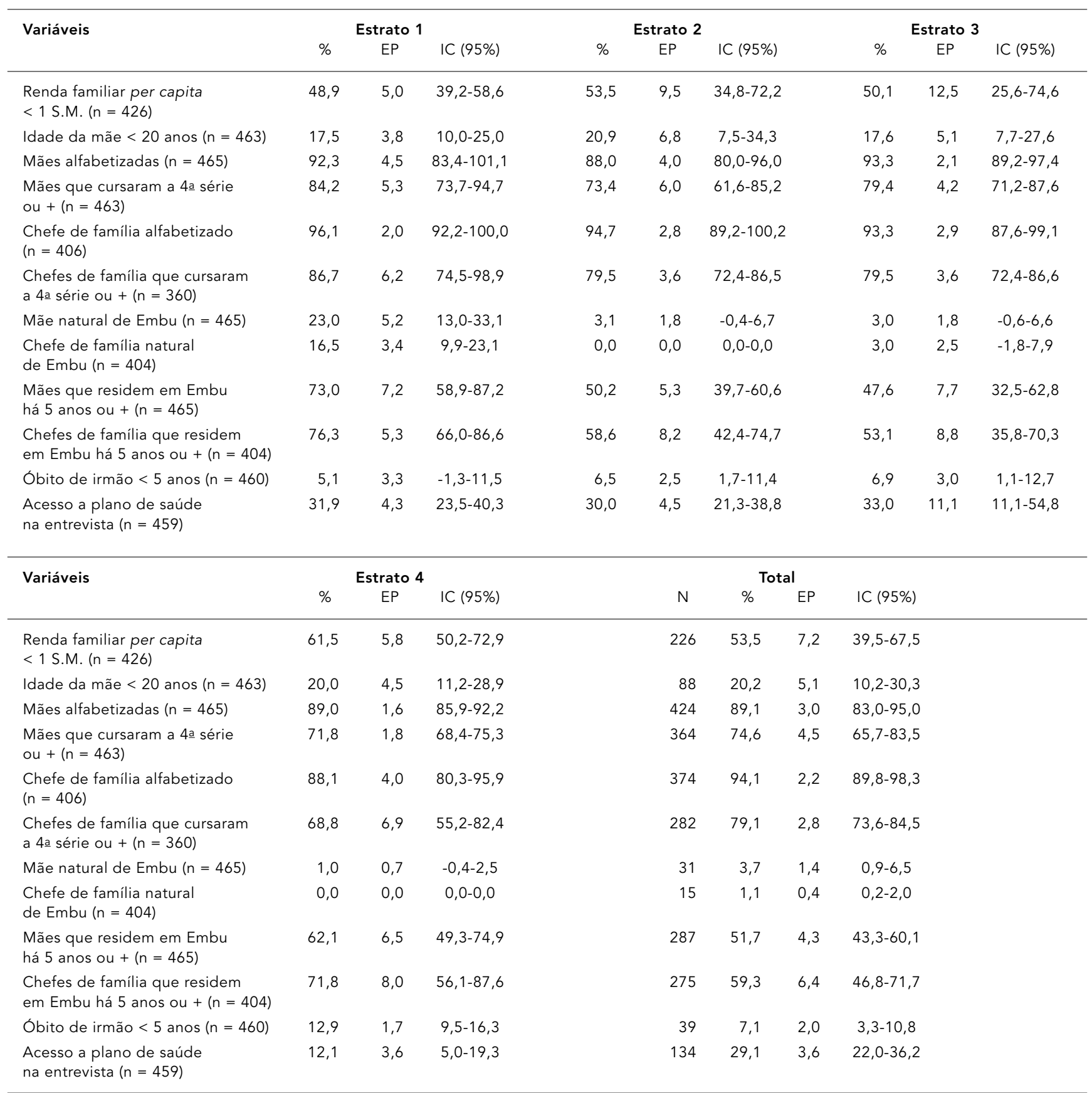

$\mathrm{n}$ = número total de questionários com informação para a variável analisada; nas proporções relacionadas ao chefe da família foram excluídos os casos em que a mãe era chefe de família.

$\mathrm{SM}=$ salário mínimo; $\mathrm{EP}=$ erro padrão estimado.

Obs: proporções estimadas com diferenças estatisticamente significantes entre estrato 1 e estratos 2, 3, 4 e total

do município para naturalidade da mãe e do chefe de família, e entre os estratos 1 e 4 quanto ao acesso a plano privado. 
Assistência pré-natal - crianças menores de um ano. Embu, São Paulo, Brasil, 1996.

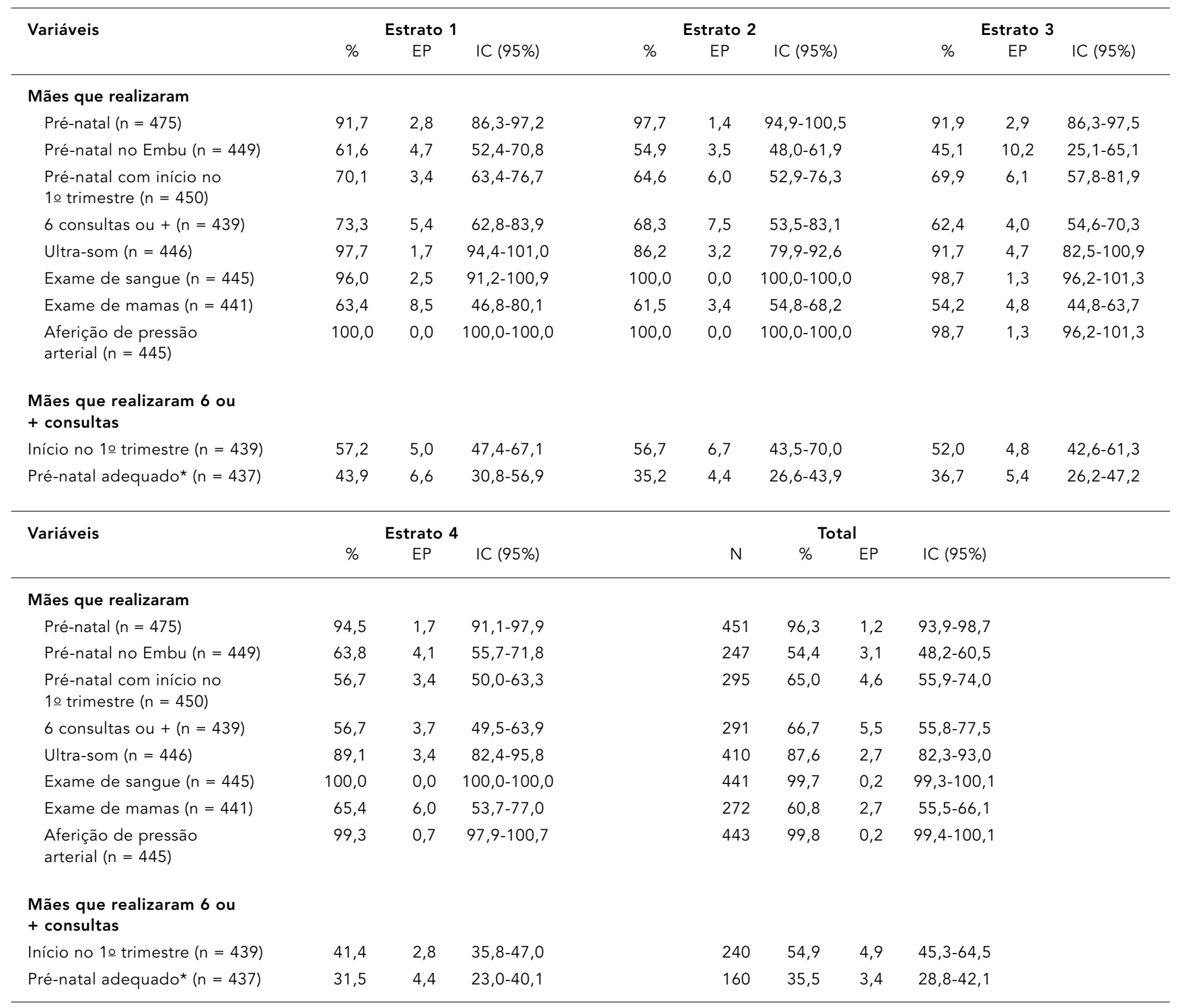

$\mathrm{n}$ = número total de questionários com informação para a variável analisada; nas proporções relacionadas ao chefe da família foram excluídos os casos em que a mãe era chefe de família.

$\mathrm{EP}=$ erro padrão estimado.

* foi considerado pré-natal adequado: início no 1ㅇ trimestre, 6 ou mais consultas, aferição de pressão arterial, coleta de exame de sangue, mamas examinadas e realização de ultra-som.

Obs: proporções estimadas com diferenças estatísticas significantes entre estratos 1 e 4 para início do pré-natal no 1 o trimestre e entre estrato 1 e estrato 2 e total do município para realização do ultra-som.

\section{Discussão}

Algumas considerações devem ser feitas para uma melhor interpretação dos resultados obtidos: (a) heterogeneidade interna dos estratos, mais acentuada no estrato 1 devido à presença de caseiros, com baixos salários, moradores de condomínios de alto padrão; (b) empreendimento imobiliário de padrão superior à média do município no estrato 3, entre o ano de 1991 (quando foi realizado o censo cujos dados foram utilizados para a estratificação) e o ano de 1996 (quando se realizou a coleta de dados deste estudo), resultando em um segmento populacional com maior acesso a planos privados e manutenção de vínculos com os serviços de saúde anteriormente utilizados. Estes fatos determinaram resultados, num primeiro momen- 
Assistência ao parto, intercorrências neonatais e peso ao nascer - crianças menores de um ano. Embu, São Paulo, Brasil, 1996.

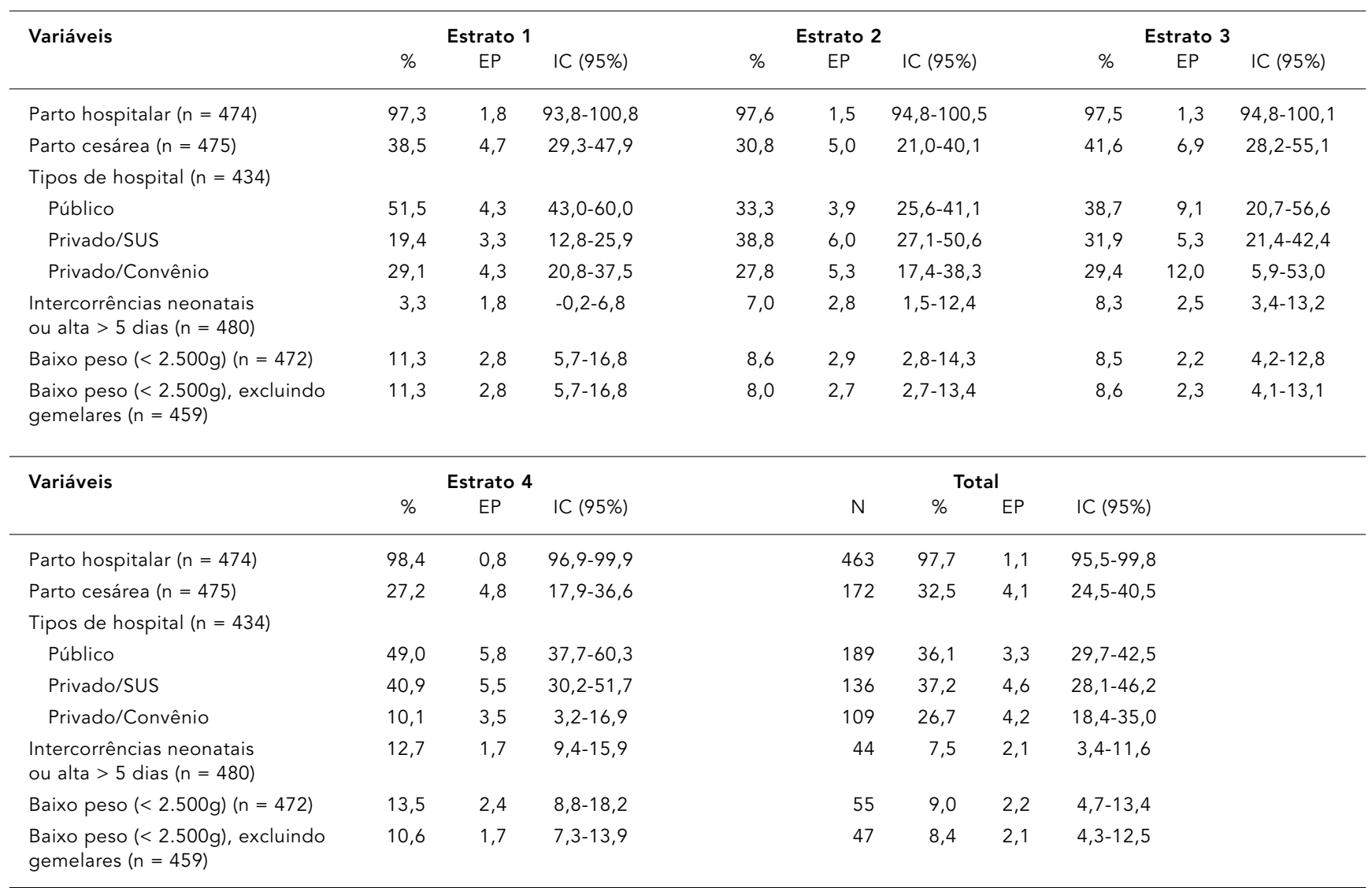

$\mathrm{n}$ = número total de questionários com informação para a variável analisada.

EP $=$ erro padrão estimado.

Obs: proporções estimadas com diferença estatisticamente significantes entre estrato 1 e estrato 2 e total

do município para parto em hospital público; entre estrato 4 e estratos 1 e total do município para parto

em hospital privado/convênio; entre estratos 1 e 4 para intercorrências neonatais.

Tabela 4

Qualidade da assistência pré-natal na Rede Básica do Embu - crianças menores de um ano. Embu, São Paulo, Brasil, 1996.

\begin{tabular}{|c|c|c|c|c|c|c|c|c|c|c|}
\hline & \multicolumn{3}{|c|}{ Adequado* } & \multicolumn{3}{|c|}{$\begin{array}{c}\text { Pré-natal } \\
\text { Não adequado }\end{array}$} & \multicolumn{4}{|c|}{ Total } \\
\hline & $\%$ & EP & IC (95\%) & $\%$ & EP & IC (95\%) & $\mathrm{N}$ & $\%$ & EP & IC (95\%) \\
\hline $\begin{array}{l}\text { Pré-natal realizado } \\
\text { no Embu }(n=436)\end{array}$ & 39,7 & 5,5 & $29,0-50,4$ & 60,3 & 5,5 & $49,6-71,0$ & 239 & 54,4 & 3,1 & $48,2-60,5$ \\
\hline $\begin{array}{l}\text { Pré-natal realizado fora } \\
\text { do Embu }(n=436)\end{array}$ & 30,6 & 6,7 & $17,5-43,7$ & 69,4 & 6,7 & $56,3-82,5$ & 197 & 45,6 & 3,1 & $39,5-51,8$ \\
\hline
\end{tabular}

Odds ratio $=0,675(0,28-1,63)$

$\mathrm{n}=$ número total de questionários com informação para a variável analisada.

$\mathrm{EP}=$ erro padrão estimado.

* foi considerado pré-natal adequado: início no 1ㅇ trimestre, 6 ou mais consultas, aferição de pressão

arterial, coleta de exame de sangue, mamas examinadas e realização de ultra-som. 
Tabela 5

Resultado da análise de regressão logística para assistência pré-natal com início após o primeiro trimestre e número de consultas inferior a seis. Embu, São Paulo, Brasil, 1996.

\begin{tabular}{lcccc}
\hline Variável & Coeficiente & Valor de p & Odds ratio & IC (95\%) \\
\hline $\begin{array}{lccc}\text { Renda familiar } \\
\text { per capita }<1 \text { SM }\end{array}$ & 0,8112 & $<0,001$ & 2,3 & $1,4-3,5$ \\
Mãe $<20$ anos & 0,7889 & 0,005 & 2,2 & $1,3-3,8$ \\
$\begin{array}{l}\text { Falta de acesso ao plano } \\
\text { privado de saúde }\end{array}$ & 0,6084 & 0,02 & 1,8 & $1,1-3,0$ \\
\hline
\end{tabular}

$\mathrm{SM}=$ salário mínimo voráveis em relação à RMSP que, em 1996, apresentou 54,5\% das gestações de nascidos vivos com seis ou mais consultas (Fundação Seade, 2001) e ao estudo de Silveira et al. (2001) na cidade de Pelotas; porém, mais desfavoráveis quando comparados ao estudo de Monteiro et al. (2000d), em São Paulo, no mesmo ano. Nesse estudo, os autores verificaram que $88,6 \%$ das mães realizaram cinco ou mais consultas e $71,6 \%$ iniciaram o pré-natal no primeiro trimestre de gestação; constataram, também, nítida desvantagem para o tercil inferior de renda. Em nosso estudo, no estrato 4, o início do pré-natal foi mais tardio e com menor número de consultas em relação ao estrato 1 e ao total do município, com diferenças estatisticamente significantes. A análise multivariada (Tabela 5), que considerou estes dois indicadores simultaneamente, demonstrou associação desta condição com a baixa renda, idade da mãe inferior a vinte anos e falta de acesso a plano privado de saúde, revelando que grupos mais vulneráveis acabam recebendo pior assistência. Entre estas mães mais jovens, concentram-se, possivelmente, aquelas sem companheiro estável e que requerem maior atenção, não só devido à maior freqüência de complicações relacionadas à gestação (Gama et al., 2001), mas pela necessidade de se estruturarem diante desta nova situação de vida e de responsabilidades.

A realização do pré-natal na rede básica do município foi referida por $54,4 \%$ das mães, confirmando os dados de cobertura estimados pela Secretaria Municipal de Saúde. No estrato 4 chegou a $63,8 \%$, refletindo a maior utilização das unidades municipais por este segmento populacional. Na primeira consulta das crianças matriculadas nas unidades básicas de saúde, as razões comumente relatadas pelas mães para a busca de outros serviços eram: ausência de referência da Rede Básica do Município para o hospital onde seria realizado o parto; ausência de vínculo entre o médico do pré-natal e o da maternidade para realização de cesariana eletiva visando à laqueadura; dificuldades para agendamento das consultas nas unidades básicas e maior conveniência devido à proximidade do local de seu trabalho. A importância da qualidade e do vínculo entre assistência pré-natal e o local do parto, referida por gestantes e mães, foi observada por outros autores (Lamy et al., 2000; Santos, 2000). Verifica-se, assim, a complexidade dessa assistência, que não deve ser restrita à gestante e a seu feto, mas sim à mulher, considerando seu contexto familiar e social, bem como suas necessidades relacionadas ao planejamento familiar após o parto. 


\section{A assistência ao parto}

Em 1996, ano de realização deste estudo, não havia leitos de maternidade em Embu e, portanto, referência e contra-referência hospitalar, considerado um importante indicador de qualidade da atenção pré e perinatal pelo MS (2000); $97,7 \%$ dos partos foram hospitalares, lembrando que esta é uma realidade de regiões urbanas do país (MS, 1997). O tipo de serviço utilizado público, privado conveniado com SUS ou privado/convênio - esteve relacionado, sobretudo, ao acesso a planos privados de saúde (cerca de $30 \%$ das famílias). A freqüência de cesarianas em nosso estudo foi acima do preconizado pela Organização Mundial da Saúde (WHO, 1985); porém, ainda menor que a constatada na RMSP, que é de $47,1 \%$ (MS, 1997), e no estudo de Monteiro et al. (2000d), que foi de $48,9 \%$. Uma das hipóteses seria o menor acesso aos serviços privados das famílias do Embu, nos quais, comprovadamente, se apresentam os mais altos índices de partos cirúrgicos (Yazlle, 2001). A diferença observada entre serviços públicos (próprios do SUS) e privados conveniados com o SUS deve ser analisada com cautela, pois os serviços públicos, em nosso estudo, estavam representados especialmente por hospitais universitários, referências para as gestantes portadoras de doenças crônicas ou de alto risco, concentrando maior proporção de cesarianas.

\section{As intercorrências neonatais}

e o peso ao nascer

As intercorrências neonatais foram mais freqüentes no estrato 4, evidenciando as conseqüências da assistência inadequada nesse segmento populacional. Vários estudos têm comprovado a importância da assistência pré-natal de qualidade na redução de complicações e óbitos nesse período (Bolzán et al., 2000; Menezes et al., 1998).

Quanto ao baixo peso ao nascer, importante indicador de saúde de uma população, tem se verificado, nos últimos anos, que as diferenças entre regiões do país e entre os grupos populacionais procedentes de diferentes classes sociais, começam a apresentar um comportamento aparentemente paradoxal. É percebida uma redução das diferenças entre as freqüências de baixo peso, observando-se, em regiões mais desenvolvidas, uma estagnação deste indicador e até mesmo um aumento (Barbieri et al., 2000; Monteiro et al., 2000c; Silva et al., 1998). As hipóteses aventadas para o fato são várias. De um lado, poderia ser o maior número de intervenções em virtude de um controle mais adequado de pré-natal e maior possibilidade de diagnós- tico precoce de situações de risco, gerando recém-nascidos prematuros. De outro, a situação poderia ser atribuída à interrupção precoce da gravidez, sem indicação, com o objetivo de realizar partos cesarianas agendados. As porcentagens de cesarianas no país, sobremodo em capitais, são alarmantes (MS, 2002).

No Embu, obteve-se uma porcentagem de baixo peso ao nascer de $9,0 \%$ considerando todas as crianças e $8,4 \%$ considerando apenas os nascimentos únicos. Estas porcentagens foram obtidas com base em uma amostra de sobreviventes, o que tende a subestimar a porcentagem real, em razão da mortalidade no período neonatal. Assim, trata-se de uma aproximação, comparável aos dados da cidade de São Paulo (Monteiro, 2000c), também de uma amostra probabilística de crianças menores de dois anos 7,9\% em 1984/1985 e 8,7\% em 1995/ 1996.

\section{Conclusões finais}

Ainda que as noções de igualdade e eqüidade sejam utilizadas indistintamente, políticas voltadas para a eqüidade pressupõem redistribuição desigual de recursos, visando a ajustes que devem ser efetuados em função de fatores biológicos, sociais e político-organizacionais (Travassos et al., 2000). Os resultados deste estudo revelaram que a procura do serviço de saúde para a assistência pré-natal e parto é praticamente universal no Embu, e a hipótese de que a população de algumas áreas, em especial as favelas, estivessem sem assistência, não foi confirmada. Verificamos, entretanto, que nesse estrato houve maiores dificuldades de acesso e deficiências quanto à qualidade da atenção, contribuindo, provavelmente, para uma maior freqüência de intercorrências neonatais, talvez também de mortalidade, aspecto este não abordado neste estudo porque considerou uma amostra de sobreviventes. Estes resultados foram apresentados e discutidos no Conselho $\mathrm{Mu}$ nicipal de Saúde, no ano de 1996, e se constituíram em importante subsídio para o aprimoramento do Programa de Assistência Pré-Natal.

$O$ reconhecimento de necessidades e do direito à saúde deve contribuir para a concretização de uma efetiva participação da população, quer em instâncias já previstas em lei (Brasil, 1990), como conselhos municipais de saúde, quer no cotidiano dos serviços. Desse exercício de cidadania, que envolve conflitos e interesses diversos, será possível a superação dessas deficiências rumo à consolidação do SUS, garantindo, de forma efetiva, a universalidade e a eqüidade na atenção à saúde. 


\section{Agradecimentos}

Pesquisa financiada pela FAPESP (Fundação de Amparo à Pesquisa do Estado de São Paulo), Processo no 95/3438-7.

\section{Referências}

BARBIERI, M. A.; SILVA, A. A. M. \& BETTIOL, H., 2000. Risk factors for the increasing trend in low birth weight among live births born by vaginal delivery, Brazil. Revista de Saúde Pública, 34:596-602.

BOLZAN, A.; GUIMAREY, L. \& NORRY, M., 2000. Factores de riesgo de retardo de crecimiento intrauterino y prematurez en dos municipios de la Provincia de Buenos Aires. Jornal de Pediatria, 76: C8-C14.

BRASIL, 1990. Lei 8.142, de 28 de dezembro de 1990. Dispõe sobre a participação da comunidade na gestão do Sistema Único de Saúde - SUS e sobre as transferências intergovernamentais de recursos financeiros na área da saúde e dá outras providências. Brasília: Diário Oficial da República Federativa do Brasil, no 249, 31 dez, pp. 25694-25695.

EMPLASA (Empresa Metropolitana de Planejamento da Grande São Paulo), 1994. Plano Metropolitano da Grande São Paulo 1994/2010. São Paulo: EMPLASA.

FONSECA, L. A. M. \& LAURENTI, R., 2000. Evolução da mortalidade materna. In: Velhos e Novos Males da Saúde no Brasil: A Evolução do País e suas Doenças (C. A. Monteiro, org.), pp.186-192, São Paulo: Editora Hucitec/Núcleo de Pesquisas Epidemiológicas em Nutrição e Saúde, Universidade de São Paulo.

FREI, F., 1997. Análise de Agrupamentos: Estudo Metodológico e Aplicações em Epidemiologia, São Paulo. Dissertação de Mestrado, São Paulo: Faculdade de Saúde Pública, Universidade de São Paulo.

FUNDAÇÃO SEADE (Fundação Sistema Estadual de Análise de Dados), 2001. Informações dos Municípios Paulistas. 25 Fevereiro 2001 <http://www. seade.gov.br>.

GAMA, S. G. N.; SZWARCWALD, C. L.; LEAL, M. C. \& THEME-FILHA, M. M., 2001. Gravidez na adolescência como fator de risco para baixo peso ao nascer no Município do Rio de Janeiro, 1996 a 1998. Revista de Saúde Pública, 35:74-80.

IBGE (Fundação Instituto Brasileiro de Geografia e Estatística), 1992. Censo Demográfico 1991. Rio de Janeiro: IBGE.

KISH, L., 1965. Survey Sampling. New York: John Wiley \& Sons.

KRAMER, M. S., 1987. Determinants of low birth weight: Methodological assessment and metaanalysis. Bulletin of the World Health Organization, 65:663-737.

LAMY, Z. C.; REINALDO, A. M. S.; FERNANDES, L. P. \& MOCHEL, E. G., 2000. O olhar das mulheres: Vivendo a gravidez e o parto. In: Avaliação da Qualidade de Maternidades (M. T. S. S. B. Alves \& A. A. M. Silva, org.), pp. 56-63, São Luís: Núcleo de Pesquisa em Saúde Materno-Infantil, Departamento de Saúde Pública, Universidade Federal do Maranhão.

MENEZES, A. M. B.; BARROS, F. C.; VICTORA, C. G., TOMASI, E.; HALPERN, R. \& OLIVEIRA, A. L. B., 1998. Fatores de risco para mortalidade perinatal em Pelotas. Revista de Saúde Pública, 32:209-216.

MONTEIRO, C. A.; BENÍCIO, M. H. \& ORTIZ, L. P., 2000c. Tendência secular do peso ao nascer na Cidade de São Paulo (1976-1998). Revista de Saúde Pública, 34(Sup. 6):26-40.

MONTEIRO, C. A.; FRANÇA Jr., I. \& CONDE, W. L., 2000d. Evolução da assistência materno-infantil na Cidade de São Paulo (1984-1996). Revista de Saúde Pública, 34(Sup. 6):19-25.

MONTEIRO, C. A.; IUNES, R. F. \& TORRES, A. M., 2000a. A evolução do país e de suas doenças: Síntese, hipóteses e implicações. In: Velhos e Novos Males da Saúde no Brasil - A Evolução do País e de suas Doenças (C. A.Monteiro, org.), pp. 349-356, São Paulo: Editora Hucitec/Núcleo de Pesquisas Epidemiológicas em Nutrição e Saúde, Universidade de São Paulo.

MONTEIRO, C. A.; MONDINI, L. \& COSTA, R. B. L., 2000b. Evolução da mortalidade infantil e do retardo de crescimento nos anos 90: Causas e impacto sobre as desigualdades regionais. In: Velhos e Novos Males da Saúde no Brasil - A Evolução do País e de suas Doenças (C. A. Monteiro, org.), pp. 393-420, São Paulo: Editora Hucitec/Núcleo de Pesquisas Epidemiológicas em Nutrição e Saúde, Universidade de São Paulo.

MS (Ministério da Saúde), 1997. Indicadores e Dados Básicos. 20 Fevereiro $2001<$ http://www.saude. gov.br/inform/IDB97>.

MS (Ministério da Saúde), 2000. Portaria no 570, Programa de Humanização no Pré-Natal e Nascimento. 1o Junho $2000<$ http:/ / www.saude.gov.br/pro gramas/mulher/prenatal.htm>.

MS (Ministério da Saúde), 2002. Indicadores e dados Básicos - Proporção de Partos Cesáreos. 29 Março 2002 <http://www.saude.gov.br>.

NEUMANN, N. A., 2000. Cobertura, Qualidade e Eqüidade da Atenção Pré-natal e ao Parto, Segundo a Forma de Pagamento destes Serviços, Criciúma (SC). Tese de Doutorado, São Paulo: Faculdade de Saúde Pública, Universidade de São Paulo.

SANTOS, I. S.; BARONI, R. C.; MINOTTO, I. \& KLUMB, A. G., 2000. Critérios de escolha de postos de saúde para acompanhamento pré-natal em Pelotas (RS). Revista de Saúde Pública, 34:603-609. 
SCHLAEPFER, L. \& INFANTE, C., 1995. Bajo peso al nacer em México: Evidencias a partir de uma encuesta retrospectiva a nível nacional. Boletín Médico do Hospital Infantil de México, 52:168-179.

SMS-EMBU (Secretaria Municipal de Saúde do Embu), 1996. Programa de Assistência ao Pré-Natal. Critérios de Risco, Normas e Procedimentos de Rotina. Embu (SP). São Paulo: SMS-Embu.

SILVA, A. A. M.; BARBIERI, M. A.; GOMES, U. A. \& BETTIOL, H., 1998. Trends in low birth weight: A comparison of two birth cohorts sparated by a 15-year interval in Ribeirão Preto, Brazil. Bulletin of the World Health Organization, 76:73-84.

SILVA, N. N.; PEDROSO, G. C.; PUCCINI, R. F. \& FURLANI, W. J., 2000. Desigualdades sociais e uso de serviços de saúde: Evidências de análise estratificada. Revista de Saúde Pública, 34:44-49.

SILVA, N. N.; PUCCINI, R. F.; ARAÚJO, N. S.; PEDROSO, G. C.; GOMES, M. T.; FURLANI, W. J. \& SOARES, F. S., 1997. Morbidade e Utilização de Serviços de Saúde no Município do Embu: Estudo Epidemiológico na Área da Saúde Infantil. São Paulo: Faculdade de Saúde Pública, Universidade de São Paulo/Departamento de Pediatria, Universidade Federal de São Paulo.
SILVEIRA, D. S.; SANTOS, I. S. \& COSTA, J. S. D., 2001. Atenção pré-natal na rede básica: Uma avaliação de estrutura e processo. Cadernos de Saúde Pública, 17:131-139.

TRAVASSOS, C.; VIACAVA, F.; FERNANDES, C. \& ALMEIDA, C. M., 2000. Desigualdades geográficas e sociais na utilização de serviços de saúde no Brasil. Ciência \& Saúde Coletiva, 5:133-149.

UNIFESP (Universidade Federal de São Paulo), 2001. Programa de Integração Docente-Assistencial do Embu, 2001. 13 Maio 2001 <http:/ /www.unifesp. br>.

WHO (World Health Organization), 1985. Apropriate technology for birth. Lancet, 2:436-437.

YAZLLE, M. E. H.; ROCHA, J. S. Y.; MENDES, M. C.; PATA, M. C.; MARCOLIN, A. C. \& AZEVEDO, G. D. 2001. Incidência de cesáreas segundo fonte de financiamento da assistência ao parto. Revista de Saúde Pública, 35:202-206.

Recebido em 17 de agosto de 2001

Versão final reapresentada em 13 de março de 2002 Aprovado em 28 de junho de 2002 